\title{
On-Wafer Measurements of Nonlinear Effects in High Temperature Superconductors
}

\author{
James C. Booth, Leila R. Vale, and Ronald H. Ono
}

\begin{abstract}
We present the results of comprehensive onwafer microwave probe station measurements of the nonlinear properties of coplanar waveguide devices patterned from hightemperature superconductor (HTS) thin films. We introduce a sequence of measurements and analysis that is designed to describe the nonlinear response of microwave devices in increasingly general terms, and that verifies the resulting description with a number of different nonlinear measurements of different patterned devices. We demonstrate the use of this methodology to analyze the nonlinear response of $\mathrm{YBa}_{2} \mathrm{Cu}_{3} \mathrm{O}_{7-8}$ (YBCO) thin films at $76 \mathrm{~K}$, and find that all of our measurements can be satisfactorily explained by a currentdependent penetration depth of the form $\lambda=\lambda_{0}\left[1+\left(\mathrm{J} / \mathrm{J}_{0}\right)^{2}\right]$. The parameter $J_{0}$ is a current-density scale that describes the strength of the material nonlinearity, and can be used as a suitable parameter for optimizing film growth techniques.
\end{abstract}

Index Terms-high-temperature superconductors, microwave devices, nonlinear response.

\section{INTRODUCTION}

$\mathrm{N}$ Tonlinear effects in high-temperature superconductor (HTS) microwave devices can cause significant problems, particularly in telecommunications systems. In applications such as receive filters, intermodulation distortion is a serious concern, since it can produce interference signals within the band of interest. In transmission-line structures such as delay lines, and for transmit applications, harmonic-generation effects can be a problem. Other nonlinear phenomena include crossmodulation and amplitude modulation to phase modulation (AM/PM) conversion [1]. The increase in insertion loss and possible device damage at elevated powers are obviously major concerns for any high-power applications.

Given the many different manifestations of nonlinearity in HTS devices, we are interested in determining the best way to characterize nonlinear effects in HTS microwave applications in order to develop a general description of nonlinear phenomena in high-temperature superconductors. Since nonlinear effects can very easily be caused by extrinsic sources (such as damaged regions [2], connectors or cables of poor quality [3], material interfaces [1]) we need to address

Manuscript received September 19,2000. This work was supported in part by the Office of Naval Research

James C. Booth is with the National Institute of Standards and Technology, Boulder, CO 80305 USA (telephone: 303-497-7900, e-mail: booth@boulder.nist.gov).

Leila R. Vale is with the National Institute of Standards and Technology, Boulder, CO 80305 USA (telephone: 303-497-5121, e-mail: vale@boulder.nist.gov).

Ronald $\mathrm{H}$. Ono is with the National Institute of Standards and Technology, Boulder, CO 80305 USA (telephone: 303-497-3762, e-mail: ono@boulder.nist.gov). whether or not it is possible or meaningful to develop a general description of the nonlinear response in HTS microwave devices. If it is possible to construct a general description, we want to use it to characterize HTS thin films so that the material with the smallest nonlinear response can be selected for use in device applications. We also want to be able to predict specific nonlinear effects, such as harmonicgeneration and/or intermodulation distortion, for a given device under arbitrary stimulus. It would also be beneficial to know the critical power or current density above which damage occurs for a specific material or device. Finally, we want to discover the origins of nonlinear effects in these materials, in order to begin to minimize them. The entire process may be accelerated by determining whether the nonlinear response observed at microwave frequencies correlates with any other material properties that are more easily measured.

In what follows we describe the application of on-wafer microwave measurements to the nonlinear characterization of HTS thin films. We use the term on-wafer measurements to describe measurements made on multiple patterned devices on the same thin-film sample through the use of movable microwave probes. By combining different patterned structures on a single HTS thin film with a number of different nonlinear measurements, we are able to systematically characterize the nonlinear response of the HTS material under test. We then illustrate the use of these onwafer measurements to characterize a specific thin-film sample, and demonstrate the successful extraction of the relevant nonlinear material properties. We show how these nonlinear material properties describe our current experimental results and can be used to predict general device response.

\section{NONLINEAR MEASUREMENTS}

Our approach to nonlinear characterization of HTS thin films is summarized in Fig. 1. We begin with a set of power-dependent measurements on a single device, in order to obtain the complex impedance as a function of power for a particular device geometry. We then proceed to generalize the nonlinear description while performing additional nonlinear measurements, initially in the same device geometry. In this manner we aim to obtain a description of the HTS thin film in terms of nonlinear material parameters, which consistently accounts for the results of our different measurements. We then use the nonlinear material parameters that we have derived to predict the nonlinear response of one or several different device geometries, and compare these predictions directly to measurements.

Typically one performs the procedure illustrated in Fig. 1 in a sequential fashion, fabricating new devices for each step. 


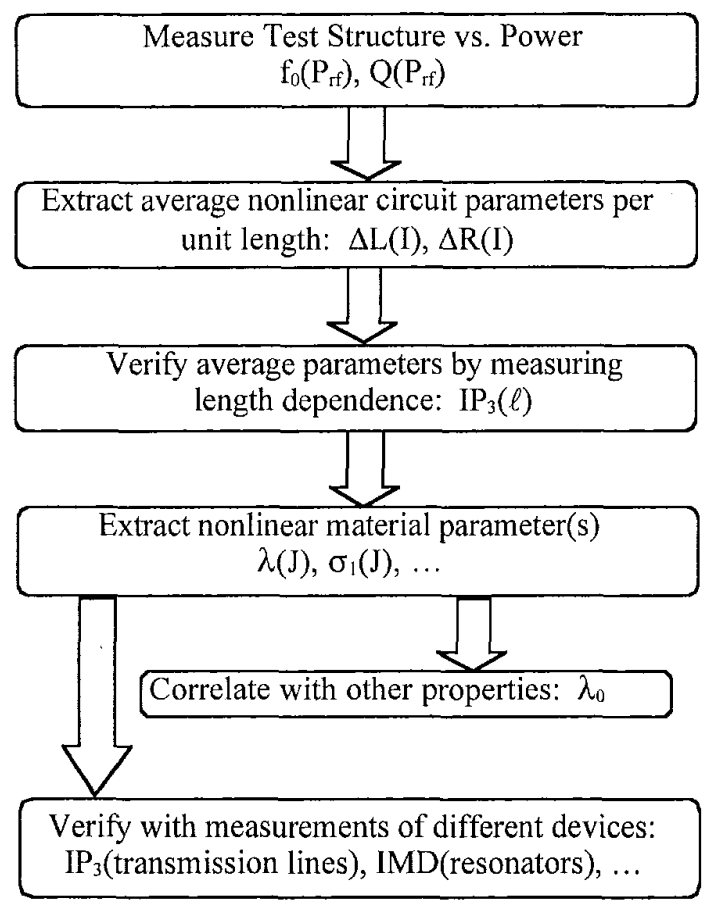

Fig.1. Schematic diagram of nonlinear measurement/analysis sequence

This has the disadvantage that one is never completely sure that the material for subsequent devices is the same as the material for the initial measurements. A considerable advantage of performing on-wafer measurements is that we are able to fabricate all the devices necessary for carrying out the measurement sequence in Fig. 1 on a single thin-film sample. In addition to substantially reducing the time required to perform the entire measurement sequence, the onwafer measurements stand a better chance of ensuring that the material for different devices is nearly identical.

We have implemented these on-wafer techniques to characterize the nonlinear response of coplanar waveguide (CPW) devices fabricated from pulsed-laser deposited YBCO thin films at $76 \mathrm{~K}$. The initial test structure is typically a CPW resonator designed to have a resonant frequency of several GHz. We measured the resonant frequency and quality factor $(\mathrm{Q})$ as a function of incident power for this structure, and analyzed the resulting data to obtain the nonlinear inductance and resistance per unit length, $\Delta \mathrm{L}(\mathrm{I})$ and $\Delta R(I)$, respectively. We then used the measured nonlinear inductance per unit length $\Delta \mathrm{L}(\mathrm{I})$ to calculate the third-order intercepts $\mathrm{IP}_{3}$ one would obtain for transmission lines of the same cross-sectional geometry as the resonator, but of variable length, and compared these results with measurements of the third-order intercepts. This step is crucial in order to determine whether the power-dependent response measured in the initial test resonator is representative of the nonlinear response of the material itself, and is not a spurious response due to damage or other extrinsic effects. Once we verified that the nonlinear response was in fact due to the material, we proceeded to extract the relevant nonlinear material parameters, which for this case is a current-density dependent penetration depth, $\lambda(\mathrm{J})$. Having determined what we believe is the underlying nonlinear material property $(\lambda(J))$, we then calculated the expected third-harmonic generation vs. power for $\mathrm{CPW}$ transmission lines of different cross-sectional geometries, and verified these predictions with measurements of devices on the same wafer as used for the previously described power-dependent resonator and third-harmonic generation measurements. Completion of the measurement sequence illustrated in Fig.1 gave us confidence that we have a general description for the nonlinear response of the material under study. We then proceeded to make meaningful comparisons between materials grown under different growth conditions, and began to look for correlations between the measured nonlinear material properties and other experimental and material parameters.

In what follows we will describe in detail how this measurement and analysis sequence has been carried out. Since many of the individual steps in Fig. 1 have been reported before, we describe in detail our recently developed calibrated resonator measurements, which provide accurate characterization of the power-dependent impedance of a patterned $\mathrm{CPW}$ resonator. We conclude by examining some of the limitations of the resulting simple description for the nonlinear response of HTS microwave devices.

\section{CALIBRATEd RESONATOR MEASUREMENTS}

Our superconducting CPW resonator was fabricated from a $400 \mathrm{~nm}$ thick YBCO film grown on a $0.5 \mathrm{~mm}$ thick $\mathrm{LaAlO}_{3}$ substrate by pulsed laser deposition [4]. The YBCO film was approximately $16 \mathrm{~mm} \times 16 \mathrm{~mm}$ in area. The resonator under test was $11.5 \mathrm{~mm}$ in length, and was coupled to two $50 \Omega$ sections of CPW transmission lines by capacitive gaps $100 \mu \mathrm{m}$ wide. The resonator had a center conductor linewidth of $21 \mu \mathrm{m}$ and a gap spacing (between center conductor and ground planes on either side) of $40 \mu \mathrm{m}$. The YBCO sample was mounted on a $0.5 \mathrm{~mm}$ thick sapphire wafer that was attached to a copper cold stage in our cryogenic microwave probe station. The fundamental mode for this resonator at $76 \mathrm{~K}$ is at a frequency of approximately $3.7 \mathrm{GHz}$ and has an unloaded $\mathrm{Q}$ of roughly 5500 . Simulations [5] of this geometry based on dielectric resonator and mutual inductance measurements, respectively, of $R_{s}$ and $\lambda$ for unpatterned companion samples verified this value for the quality factor.

Included along with this resonator on our $\mathrm{YBCO}$ thin film sample were patterned calibration artifacts, which are CPW transmission lines of the same cross-sectional dimensions as the resonator, and which include a short-circuit reflect, a through line, and transmission lines of four different lengths. Prior to measuring the scattering parameters (S-parameters) of the resonator, we performed a multiline through-reflect-line (TRL) calibration [6] using these calibration artifacts. This has the effect of placing the measurement reference plane onchip at the location of the resonator coupling gaps. The calibrated S-parameter measurements are therefore relative to the characteristic impedance of the CPW transmission lines, which has been shown to be very close to $50 \Omega$ and real at the resonant frequency quoted above [7]. The resonator coupling was symmetric and weak, with an insertion loss on resonance of $43.6 \mathrm{~dB}$. The value for the insertion loss is important in determining the coupling coefficients, which in 
turn are used to calculate the currents in the resonator from the incident power. The weak coupling also implies that the unloaded $\mathrm{Q}$ is given approximately by the measured $\mathrm{Q}$. It should be noted that separate TRL calibrations must be performed for each different source power range used in the measurement.

In order to adequately determine the power dependence of our CPW resonator, we needed incident powers on the order of $100 \mathrm{~mW}$. It was therefore necessary to use an external amplifier to increase the network analyzer's available power (which is typically on the order of $1 \mathrm{~mW}$ ). In order to perform the calibrations described above, we needed to measure all four S-parameters of the resonator under test with an amplifier inserted in the signal path. We used a specially modified high-power test set [8] that makes the network analyzer signal available at the source, where an external amplifier can be inserted. The reference signal was then coupled off before the main signal was fed back into the network analyzer. Fig. 2 is a simplified diagram of the modified test set. In the high-power mode shown in Fig. 2 source powers up to $20 \mathrm{~W}$ can be used. If the external amplifier is switched out of the circuit, the isolators are replaced by jumpers, and the reference-select switch is set to internal, then the network analyzer functions in low-power mode, which is similar to standard network-analyzer test sets but with slightly reduced dynamic range due to the extra signal path introduced. Use of this modified test set along with the on-wafer TRL calibrations allows us to make accurate, calibrated S-parameter measurements of our patterned CPW resonator at high powers.

Fig. 3 shows the measured power dependence of the resonant frequency and quality factor for our YBCO resonator at $76 \mathrm{~K}$ for three different ranges of source power. Recall that separate calibrations are needed for each different power

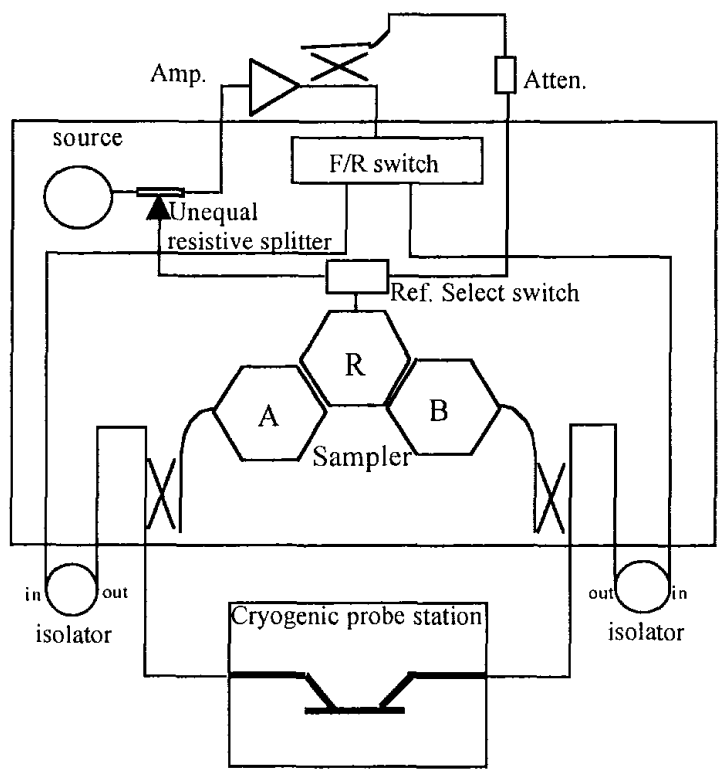

Fig. 2. Schematic diagram of network analyzer test set modified to provide high powers.
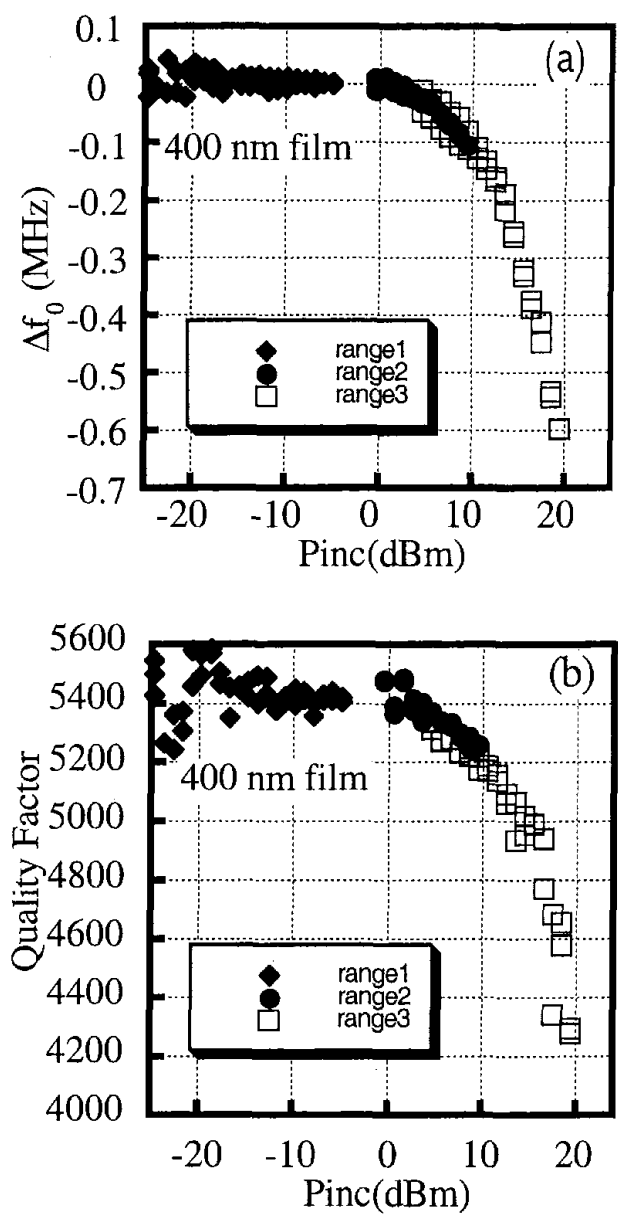

Fig. 3. Power dependence at $76 \mathrm{~K}$ and $3.7 \mathrm{GHz}$ of the (a) resonant frequency and (b) quality factor for a CPW resonator fabricated from a $400 \mathrm{~nm} \mathrm{YBCO}$ thin film.

range. The power range labeled range $1(-25$ to $-5 \mathrm{dBm}$ at the location of the probe station) is the standard test-set configuration with the amplifier switched out of the circuit. Power ranges $2(0$ to $+10 \mathrm{dBm})$ and $3(+5$ to $+20 \mathrm{dBm})$ both use the high-power test-set configuration illustrated in Fig. 2. We determined the resonant frequency and $Q$ by using the arctangent fit to the phase of the complex transmission coefficient data [9].

\section{ANALYSIS OF POWER DEPENDENT DATA}

We used the data in Fig. 3 to determine the nonlinear contribution to the resistance per unit length and inductance per unit length. If we write the total resistance per unit length as a sum of linear and nonlinear terms $R(I)=R_{0}+\Delta R(I)$, and similarly for the inductance per unit length $\mathrm{L}(\mathrm{I})=\mathrm{L}_{0}+\Delta \mathrm{L}(\mathrm{I})$, we can calculate [7] $\Delta \mathrm{R}$ and $\omega \Delta \mathrm{L}$ from the change in resonant frequency $f_{0}$ and quality factor $Q$ :

$$
\begin{aligned}
\omega \Delta L & \approx-2 \omega L_{0}\left(\frac{\Delta f_{0}}{f_{0}}\right) \\
\Delta R & \approx \omega L_{0} \Delta\left(\frac{1}{Q}\right) .
\end{aligned}
$$

In Eq. (1) we have made the assumption that the nonlinear contribution to the total inductance is small so that $\mathrm{L} \approx \mathrm{L}_{0}$. 
We can calculate the average rf current flowing in the resonator from the following expression [7]:

$$
I_{\text {avg }}=\frac{2}{\pi} \sqrt{\frac{r_{V}\left(1-r_{V}\right) 8 Q_{c} P}{n \pi Z_{0}}}
$$

where $Q$ is the unloaded quality factor, $Z_{0}$ is the characteristic impedance, $P$ is the incident power, and $r_{V}$ is the voltage insertion ratio, which is related to the insertion loss IL by $\mathrm{IL}=-20 \log \left(\mathrm{r}_{V}\right)$.

Fig. 4 shows the nonlinear contribution to the inductive reactance and resistance as a function of average current in the resonator determined from the data in Fig. 3. The solid line is a quadratic fit to the form $\omega \Delta \mathrm{L}(\mathrm{I})=\omega \mathrm{L}^{\prime} \mathrm{I}^{2}$, and gives for the parameter $\omega \mathrm{L}^{\prime}=134 \Omega /\left(\mathrm{A}^{2} \mathrm{~m}\right)$. Note that the $\Delta \mathrm{R}(\mathrm{I})$ term is much smaller than $\omega \Delta \mathrm{L}$ for almost the entire range of current values. We will use this fact below to simplify the subsequent calculations by focusing on the behavior of the nonlinear inductive reactance and neglecting the nonlinear resistance.

\section{THIRD-HARMONIC GENERATION RESULTS}

In calculating the inductive reactance per unit length above, we have made the implicit assumption that the powerdependent response measured on our $\mathrm{CPW}$ resonator represents the average response of the entire line. That is, we assume that the measured response displayed in Fig. 3 is not due to an isolated defect or any other extrinsic source. The natural way to check this assumption is to measure the nonlinear inductive reactance as a function of length to see if one obtains the expected linear dependence. This is difficult to do at the same frequency using a resonator geometry, so we make use of CPW transmission lines that have the same cross-sectional geometry as the resonator studied above. We overcome the sensitivity limitations imposed by the nonresonant transmission line geometry by measuring a different manifestation of nonlinearity, third-harmonic generation. If the nonlinearity is an inductive reactance that depends quadratically on the current, we expect [10] to observe a third-harmonic signal that increases as the third power of the incident signal, with an intercept determined by the value of $\omega \mathrm{L}^{\prime}$. We obtain [7] the following simple relationship between the so-called third-order intercept point and the parameter $\omega \mathrm{L}^{\prime}$ :

$$
\log I P_{3}=\log \frac{2 Z_{0}^{2}}{\omega L^{\prime} \ell}
$$

where $\ell$ is the transmission line length and $Z_{0}$ its characteristic impedance. Note the explicit appearance of the transmission line length $\ell$ in Eq. (3). A comparison of the experimentally determined values for the third-order intercept with the length dependence predicted by Eq. (3) thus constitutes a simple check on the assumption that the power dependence observed in our resonator measurements can be attributed to a distributed inductive reactance per unit length.

Fig. 5 displays the third-order intercept points obtained by measuring third-harmonic generation [10] on four transmission lines of different lengths (we used the different length transmission lines that make up our TRL calibration set for these measurements). The solid line is a fit to the length dependence predicted by Eq. (3). In fitting the data in

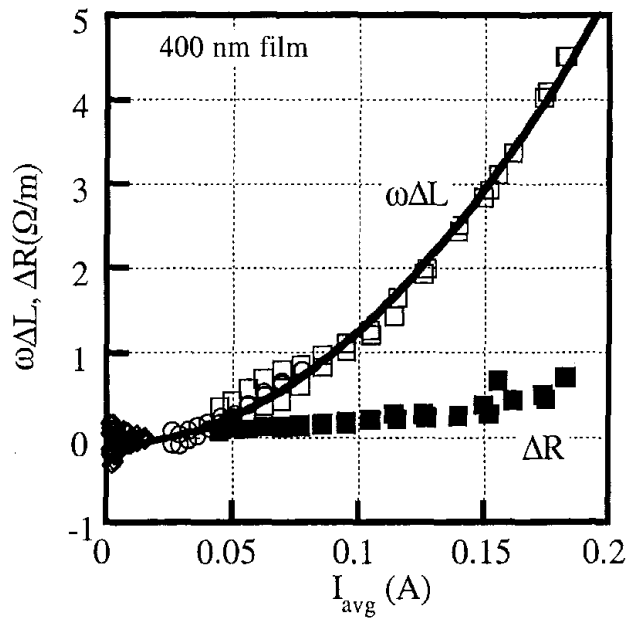

Fig. 4. Current dependence of the nonlinear contribution to the inductive reactance (open symbols) and resistance (filled symbols) for a YBCO resonator at $76 \mathrm{~K}$ and $3.7 \mathrm{GHz}$. Different plotting symbols correspond to different power ranges. The nonlinear resistance is shown for only the highest power range (squares)

Fig. 5 we allowed for the existence of an offset length, since we are interested only in differences in line length, and our transmission lines contain tapered sections to convert from the transmission-line geometry to a probe-compatible geometry. It is therefore difficult to determine the correct absolute length for the transmission lines, but the relative lengths are well determined. This figure demonstrates good agreement with the length dependence predicted by Eq. (3) and thus validates our assumption that the nonlinear reactance can be described by a distributed nonlinear reactance per unit length. In addition, we can also extract a value for the parameter $\omega \mathrm{L}^{\prime}$ from the fit in Fig. (5). We obtained $\omega L^{\prime}=198 \Omega /\left(\mathrm{A}^{2} \mathrm{~m}\right)$, which is the same order of magnitude as the value obtained from Fig. $4\left(\omega \mathrm{L}^{\prime}=134 \Omega /\left(\mathrm{A}^{2} \mathrm{~m}\right)\right.$ ). Note that it is not possible to extract the shape of $\omega \Delta \mathrm{L}(\mathrm{I})$

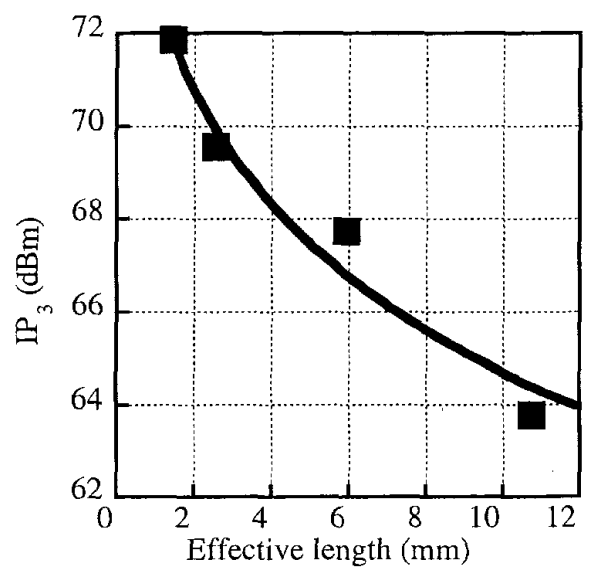

Fig. 5. Third order intercepts measured at $76 \mathrm{~K}$ for $\mathrm{CPW}$ transmission lines of different lengths at a fundamental frequency of $3.7 \mathrm{GHz}$. The transmission line cross section is the same as for the resonator analyzed previously. The solid line is a fit to the length dependence predicted by Eq.(3). 
from the harmonic generation experiments alone; we can merely say that the third-harmonic data are consistent with an assumed form for $\omega \Delta \mathrm{L}(\mathrm{I})$.

\section{NONLINEAR MATERIAL PARAMETERS AND VERIFICATION}

Given the shape of the nonlinear inductive reactance versus current curve in Fig. 4, it is reasonable to posit that such a curve can be caused by a penetration depth that varies quadratically with the current density:

$$
\lambda^{2}(T, J)=\lambda^{2}(T)\left[1+\left(\frac{J}{J_{0}(T)}\right)^{2}\right] .
$$

This form for a nonlinear penetration depth has been suggested by other authors [11] and is what one expects of the change in penetration depth due to current-induced depairing. The parameter $J_{0}$ is a current density scale and describes the strength of the material-dependent nonlinearity (larger values for $J_{0}$ imply smaller nonlinear response). The nonlinear inductive reactance $\omega \Delta \mathrm{L}(\mathrm{I})$ can be calculated from $\lambda(J)$ if the current density is known. We use a numerical calculation [12] to determine the current density for our CPW transmission lines and resonators, and use mutualinductance measurements to determine $\lambda(76 \mathrm{~K}, \mathrm{~J}=0)$. This calculation allows us to calculate $\omega \Delta L$ (I) from Eq. (4) and gives the following numerical relation [10] between the parameter $\omega \mathrm{L}^{\prime}$ and the current density scale $J_{0}$ :

$$
L^{\prime}=\frac{\mu_{0} \lambda^{2}(T) \Gamma^{\prime}}{J_{0}^{2}}
$$

where $\Gamma^{\prime}$ is a geometrical factor calculated from the numerically determined current density $\mathrm{J}$ according to

$$
\Gamma^{\prime}=\frac{\int J^{4} d S}{\left(\int J d S\right)^{4}}
$$

Using the values for $\omega \mathrm{L}^{\prime}$ determined from both the powerdependent resonator data (Fig. 4) and the data for third harmonic vs. length (Fig. 5), we obtain the following values for the nonlinear scaling-current density: $J_{0}=44.0 \mathrm{MA} / \mathrm{cm}^{2}$ (power dependence), and $\mathrm{J}_{0}=36.2 \mathrm{MA} / \mathrm{cm}^{2}$ (third-harmonic generation). Noting that these quantities were derived from very different experiments, the agreement is quite good.

The final step in our process for characterizing the nonlinear response of HTS films is to verify the nonlinear material parameter $\mathrm{J}_{0}$ for different device geometries. We also include sets of transmission lines that have wider center conductors (one set of transmission lines with center linewidth of $53 \mu \mathrm{m}$ and one set with center conductor of $105 \mu \mathrm{m})$ on our patterned chip. Repeating the above analysis of the third-order intercept data for the $53 \mu \mathrm{m}$ linewidth transmission lines yields $\mathrm{J}_{0}=51.3 \mathrm{MA} / \mathrm{cm}^{2}$, which is in the same range as the results obtained for the $21 \mu \mathrm{m}$ linewidth transmission lines and resonators. The third-harmonic analysis failed for the transmission lines of $105 \mu \mathrm{m}$ linewidth, as the measured third-order intercepts increased slightly with increasing length of transmission line rather than decreasing. This could indicate that the tapered sections of transmission line may be dominating the thirdharmonic response for these devices. Even with the failure of the $105 \mu \mathrm{m}$ transmission lines, we consider the verification step successful based on the agreement obsserved for the 53 $\mu \mathrm{m}$ transmission lines. The responses of the widest lines could however signify that there is some other contribution to the nonlinear response that is becoming important.

\section{CONCLUSIONS}

We have developed an on-wafer approach to the characterization of the nonlinear microwave response of HTS thin films. In addition to substantially increasing the number of devices that can be characterized on a given thinfilm sample, the on-wafer techniques also have the potential to yield increased measurement accuracy, as demonstrated with the calibrated power-dependent measurements described here. We find that for the device geometries considered here, a current-dependent penetration depth describes the results of a number of different measurements. For YBCO films fabricated by pulsed-laser deposition, we obtain a value for the nonlinear scaling current density of approximately $40 \mathrm{MA} / \mathrm{cm}^{2}$. We expect the measurement/analysis sequence described here to be of further use for optimizing HTS materials for reduced nonlinear response and for exploring the role of the nonlinear resistance in the different manifestations of nonlinearity in HTS microwave devices.

\section{ACKNOWLEDGMENT}

We acknowledge J.A. Beall and D.A. Rudman for providing helpful comments on the manuscript.

\section{REFERENCES}

[1] S. A. Maas, Nonlinear Microwave Circuits (Artech House, Boston, 1988), pp. 170-172.

[2] James C. Booth, et al., "Third-order harmonic generation in hightemperature superconducting coplanar waveguides at microwave frequencies," Appl. Superconduct. vol. 5, pp. 379-384,1998.

[3] Jeffrey A. Jargon, Donald C. DeGroot, and Kristopher L. Reed, "NIST passive intermodulation measurement comparison for wireless base station equipment," 52nd ARFTG Conference Digest, pp. 128$139,1998$.

[4] James C. Booth et al., "Simultaneous optimization of the linear and nonlinear microwave response of YBCO films and devices," IEEE Trans. Appl. Supercond. vol. 9, pp. 4176-4180, 1999.

[5] James C. Booth and C.L. Holloway, "Conductor loss in superconducting planar structures: calculation and measurements," IEEE Trans. Microwave Theory Tech. vol. 47, pp. 769-774, 1999.

[6] R.B. Marks, "A multiline method of network analyzer calibration," IEEE Trans. Microwave Theory Tech vol. 39, pp. 1205-1215, 1991.

[7] James C. Booth, L.R. Vale, R.H. Ono, and J.H. Claassen, "Powerdependent impedance of high temperature superconductor thin films: relation to harmonic generation," J. Supercond., submitted for publication.

[8] C. Wilker, Z.Y. Shen, P.S.W. Wang, C.F. Carter III, and J. Wineman, "Full 2-port calibrated S-parameter measurements up to $30 \mathrm{~W}$ of high temperature superconducting filters," 47th ARFTG Conference Digest, pp. 32-41, 1996.

[9] For a detailed description of this and other methods for determining resonator Q, see P.J. Petersan and S.M. Anlage, "Measurement of resonant frequency and quality factor of microwave resonators: comparison of methods,"J. Appl. Phys. vol. 84, pp. 3392-3402, 1998.

[10] James C. Booth, J.A. Beall, D.A. Rudman, L.R. Vale, and R.H. Ono, "Geometry dependence of nonlinear effects in high temperature superconducting transmission lines at microwave frequencies," J. Appl. Phys. vol. 86, pp. 1020-1027, 1999.

[11] T. Dahm and D.J. Scalapino, "Theory of intermodulation in a superconducting microstrip resonator," J. Appl. Phys. Vol. 81, pp. 2002-2009, 1997.

[12] D.M. Sheen., S.M. Ali, D.E. Oates, R.S. Withers, and J.A. Kong, "Current distribution for superconducting strip transmission lines," IEEE Trans. Appl. Superconductivity vol. 1, pp.108-115, 1991. 